3 The Ethics Committee of the American Society for Reproductive Medicine. Financial incentives in recruitment of oocyte donors. Fertil Steril 2000; 74: 216-17.

4 Caligana C, Navarro J, Vargas G, Simón C, Pellicer A, Remoht J. The effect of repeated controlled ovarian stimulation in donors. Hum Reprod 2001; 16: 2320-23.

\section{HER2 and proliferation of wound-induced breast carcinoma}

Sir-We agree with Elda Tagliabue and colleagues (Aug 16, p 527) ${ }^{1}$ that overexpression of HER2 by breast carcinoma cells has a role in their postoperative growth stimulation. However, we believe the interpretation of their results is incomplete, since angiogenesis was not considered as a mechanism of disease.

Results of numerous studies suggest that tumour progression and development of metastases in breast cancer are dependent on angiogenesis, and that HER2 amplification is closely associated with increased angiogenesis and expression of vascular endothelial growth factor (VEGF). ${ }^{2}$ The blocking antibody against HER2, trastuzumab, inhibits VEGF expression and tumour cell growth. ${ }^{3}$ Angiogenesis might also play a part in physiological processes, involving tissue repair. ${ }^{3}$ VEGF, angiopoietin, fibroblast growth factor, and transforming growth factor $\beta$ are among the most potent angiogenic cytokines in wound angiogenesis. ${ }^{4}$ Finally, the stimulatory effect of trauma on angiogenesis could explain why dormant metastatic tumour cells proliferate after surgery. The signal for this action seems to be the release of angiogenic peptide factors in response to wounding at the primary and secondary surgical insults. Enhanced postoperative angiogenesis and micrometastatic proliferation might also be due to resection of the primary tumour and removal of its inhibitory effect. ${ }^{5}$

*Mesut Tez, Erdal Göçmen,

Tayfun Özçelik

*Numune Training and Research Hospital, 5th Surgery Clinic, Sihhiye, Ankara 06100, Turkey (MT, EG); Ayhan Sahenk Foundation and Bilkent University, Department of Molecular Biology and Genetics, Bilkent, Ankara (TÖ) (e-mail: tozcelik@fen.bilkent.edu.tr)

1 Tagliabue E, Agresti R, Carcangiu ML, et al. Role of HER2 in wound-induced breast carcinoma proliferation. Lancet 2003; 362: 527-33.

2 Kumar R, Yarmand-Bagheri R. The role of HER2 in angiogenesis. Semin Oncol 2001; 28 (suppl 16): 27-32.

3 Drixler TA, Vogten MJ, Ritchie ED, et al. Liver regeneration is an angiogenesis- associated phenomenon. Ann Surg 2002; 236: 703-12.

4 Li J, Zhang YP, Kirsner RS. Angiogenesis in wound repair: angiogenic growth factors and extracellular matrix. Microsc Res Tech 2003; 60: 107-14.

5 Kell MR, Winter DC, O'Sullivan GC, Shanahan F, Redmond HP. Biological behaviour and clinical implications of micrometastases. Br f Surg 2000; 87: 1629-39.

\section{Authors' reply}

Sir-Angiogenesis is a key process in the metastatic progression of breast carcinomas, and metastases cannot develop without new vessel formation. However, vascularisation is necessary but not sufficient, since proliferation of tumour cells is also needed. HER2 is an important oncogene in breast carcinoma pathology, because its signaling pathway increases both proliferation and angiogenesis through induction of VEGF production. ${ }^{1}$ After surgery, angiogenesis can be increased directly by endothelial growth factors produced during the healing process and acting on endothelial cells in the vicinity of occult metastatic foci, and indirectly by VEGF released through HER2 activation on tumour cells. Accordingly, we noted that all postsurgical drainage fluids tested stimulated proliferation not only of tumour cells through epithelial growth factors, but also of human umbilical vein endothelial cells through endothelial growth factors. Moreover, induction of proliferation of both cell types by different drainage fluids displayed a similar extent of heterogeneity, suggesting that the degree of endothelial growth factor, like the degree of epithelial growth factor, is related to the extent of surgery as well as to the age of the patient. $^{2}$

The observation that HER2positive breast carcinomas, unlike their HER2-negative counterparts, show a high peak of early recurrence after surgery ${ }^{3}$ raises the possibility that epithelial growth factors that act on HER2-positive tumour cells underlie the stimulation of metastatic growth, since HER2 activation stimulates both proliferation and angiogenesis. Alternatively, as suggested by Mesut Tez, endothelial growth factors directly released from the wound could play the major part, with a higher likelihood of angiogenic stimulation in HER2-positive occult metastatic lesions related, for example, to a higher prevalence of endothelial cells, consistent with a role for HER2 in inducing angiogenesis. ${ }^{4}$ Also possible is that the greater likelihood of HER2-positive than HER2-negative tumour cells to exhibit heightened invasiveness and metastatic potential ${ }^{5}$ leads to growth stimulation by angiogenesis induction of a greater number of occult metastatic foci present in patients at the time of surgery. In both instances, if angiogenesis induced by endothelial growth factors released during wound healing is the most relevant event for induction of disease recurrence, the use of trastuzumab to block HER2 activity might not be effective in preventing relapses. A clinical trial with perioperative trastuzumab treatment is ongoing to address this issue.

Metastatic growth is a multistep process, but identification of one key event might be sufficient to develop specific therapeutic modalities. Independent of the actual growth factors involved in stimulating breast carcinoma recurrences, surgical intervention limited only to the extent deemed mandatory might help in the control of HER2-positive metastatic spread.

This work is support in part by the Associazione Italiana per la Ricerca sul Cancro

*Sylvie Ménard, Roberto Agresti, Manuela Campiglio, Andrea Balsari, Elda Tagliabue

* Molecular Targeting Unit, Department of Experimental Oncology (SM, MC, ET) and General Surgical B-Breast Unit (RA), Istituto Nazionale Tumori, Milan 20133, Italy; Institute of Pathology, University of Milan, Milan (AB) (e-mail: sylvie.menard@istitutotumori.mi.it)

1 Laughner E, Taghavi P, Chiles K, Mahon PC, Semenza GL. HER2 (neu) signaling increases the rate of hypoxiainducible factor 1alpha (HIF-1alpha) synthesis: novel mechanism for HIF-1 mediated vascular endothelial growth factor expression. Mol Cell Biol 2001; 21: 3995-4004.

2 Tagliabue E, Agresti R, Ghirelli C, Morelli D, Ménard S. The early relapse of premenopausal patients after surgery for node-positive breast carcinoma. Breast Cancer Res Treat 2001; 70: 155-56.

3 Ménard S, Balsari A, Casalini P, et al. HER2-positive breast carcinomas as a particular subset with peculiar clinical behaviors. Clin Cancer Res 2002; 8: 520-25.

4 Kumar R, Yarmand-Bagheri R. The role of HER2 in angiogenesis. Semin Oncol 2001; 28: 27-32.

5 Xu FJ, Stack S, Boyer C, et al. Heregulin and agonistic anti-p185(c-erbB2) antibodies inhibit proliferation but increase invasiveness of breast cancer cells that overexpress p185(c-erbB2): increased invasiveness may contribute to poor prognosis. Clin Cancer Res 1997; 3: 1629-34

Sir-Elda Tagliabue and colleagues' report ${ }^{1}$ emphasises the complexity of cell-growth regulatory mechanisms involved in breast cancer but, in our 